\title{
Mit 5G zur smarten Produktion
}

In einer Zeit hoher Volatilität setzen kürzere Markt- und Produktlebenszyklen produzierende Unternehmen auf der ganzen Welt weiter unter Druck. Um die Wettbewerbsfähigkeit zu erhalten, müssen Effizienzsteigerungen durch immer neue Prozessinnovationen erzielt werden. 5G und IoT sind ein Schlüssel zur Ermöglichung von Innovationen in der Produktion.

Herstellern und Telekommunikationsnetzbetreibern bieten 5G-Netze die Möglichkeit, intelligente Fabriken zu gestalten und dabei Technologien wie Automatisierung, Künstliche Intelligenz, Augmented Reality und das Internet der Dinge effektiv zu nutzen. Moderne Sensorik kann zwar Daten von Maschinen und Anlagen bereits sehr umfassend erheben, allerdings erfolgt die Speicherung und Analyse meist noch dezentral und zeitversetzt. Integrierte Sensorik, drahtlos über 5G-Mobilfunkverbindungen an cloudbasierte Analysetools angebunden, kann produzierenden Unternehmen jeder Größe und Branche in Zukunft dabei helfen, wertvolle Daten zu gewinnen und zu brauchbaren Informationen zu verarbeiten.

Die 5G-Technologie ermöglicht eine höhere Flexibilität, geringere Kosten und kürzere Vorlaufzeiten für die Neukonfiguration der Produktion in der smarten Fabrik. Durch den Einsatz von $5 \mathrm{G}$ zur Bewältigung der wichtigsten Herausforderungen bei der Digitalisierung für Branchen wie das verarbeitende Gewerbe können Telekommunikationsnetzbetreiber mehr als nur Netzwerkentwickler sein. Sie können zu Service-Enablern und Service-Entwicklern werden und damit neue Einnahmequellen erschließen. Welche konkreten Geschäftsmodelle $5 \mathrm{G}$ den produzierenden Unternehmen, Zulieferern und Mobilfunkbetreibern bietet, um nun die industrielle Fertigung kabellos mit $5 \mathrm{G}$ zu vernetzen, ist ein spannendes Forschungsgebiet. Wahrscheinlich wird dies zukünftig deutlich an Bedeutung gewinnen, da mit großer Sicherheit davon auszugehen ist, dass mit $5 \mathrm{G}$ nicht das Ende der Fahnenstange erreicht sein wird. In wenigen Jahren werden die schon darauffolgenden Generationen zur kommerziellen Verfügung stehen und damit auch noch viel mehr Möglichkeiten für technologische Innovationen und neue Geschäftsmodelle.

Ihre ZWF-Herausgeber und-Redaktion

Data Analytics in der Produktionstechnik

Aufgrund der stetig voranschreitenden Vernetzung produktionstechnischer Anlagen besteht heute die Möglichkeit, auf eine Vielzahl von Daten zuzugreifen und diese für unterschiedliche Anwendungsfälle, wie z.B. der Zustandsüberwachung, der Prozessregelung, der Auftragsplanung oder der Qualitätskontrolle, mittels „Data Analytics“ zu nutzten. Um junge Ingenieur:innen genau darin auszubilden, hat das Institut für Werkzeugmaschinen und Betriebswissenschaften (iwb) der Technischen Universität München im Sommersemester 2020 die digitale Master-Vorlesung „Artificial Intelligence in Production Engineering“ eingeführt. Die Vorlesung wird im Laufe des aktuellen Jahres auch in Form einer dreitägigen, anwendungsnahen OnlineSchulung für erfahrene Ingenieur:innen aus der Industrie angeboten. Für weitere Informationen wenden Sie sich bitte an Data-Analytics@iwb.tum.de.

\section{Kontakt}

Thomas Semm, M.Sc. Institut für Werkzeugmaschinen und Betriebswissenschaften

Technische Universität München Boltzmannstraße 15

85748 Garching b. München

Tel.: +49/89/289 15506 E-Mail: thomas.semm@iwb.tum.de

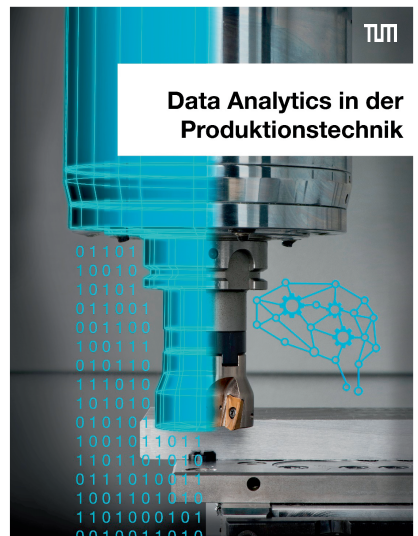

TाI . 